\title{
Management of people with early or very early stage hepatocellular carcinoma: an attempted network meta-analysis
}

\author{
Avik Majumdar ${ }^{1}$, Davide Roccarina ${ }^{1}$, Douglas Thorburn ${ }^{1}$, Brian R Davidson², \\ Emmanuel Tsochatzis ${ }^{1}$, Kurinchi Selvan Gurusamy² \\ 1Sheila Sherlock Liver Centre, Royal Free Hospital and the UCL Institute of Liver and Digestive \\ Health, London, UK \\ ${ }^{2}$ Department of Surgery, Royal Free Campus, UCL Medical School, London, UK
}

Background and Aims: Several treatment approaches exist for early stage hepatocellular carcinoma ( $\mathrm{HCC}$ ). We aimed to assess the benefits and harms of different treatment modalities for Barcelona Clinic Liver Cancer (BCLC) stage 0 (very early) and A (early) HCC by performing a network meta-analysis (NMA) using Cochrane methodology.

Methods: The Cochrane Central Register of Controlled Trials, MEDLINE, EMBASE, Science Citation Index Expanded, WHO International Clinical Trials Registry Platform and randomised clinical trials (RCTs) registers were searched to identity 6033 trials. Only RCTs with BCLC stages 0 or A HCC were included. RCTs were excluded if participants had previously undergone liver transplantation or if technical variations of the same modality were compared. Two review authors independently identified trials and extracted data. The primary outcome was mortality at maximal follow-up. We were unable to perform the intended NMA due to lack of comparative data. Therefore, we calculated the odds ratio $(\mathrm{OR})$, rate ratio $(\mathrm{RR})$, or hazard ratio $(\mathrm{HR})$ with $95 \%$ confidence intervals $(\mathrm{Cl})$ based on an available-case analysis.

Results: 3126 participants from 18 RCTs were suitable for inclusion with a follow-up of 6-42 months, during which $30 \%$ died. Most deaths occurred after 3 years of follow-up. The quality of included RCTs was generally low. Compared to radiofrequency ablation (RFA), higher mortality was seen with percutaneous acetic acid injection (PAI, HR 1.77, $\mathrm{Cl}$ 1.12-2.79) and percutaneous alcohol injection (PEI, HR 1.49, Cl 1.118- .88). No difference in mortality at maximal follow-up was observed in RFA vs surgery, or any other comparisons between treatments. In non-surgical candidates, PEI resulted in higher cancer-related mortality (OR 2.18, $\mathrm{Cl}$ 1.22-3.89) and distal/local HCC recurrence (OR 1.56, $\mathrm{Cl}$ 1.02-2.45) than RFA. Participants who had surgery had lower distal/local HCC recurrence (OR 0.52 , $\mathrm{Cl} 0.35-0.78$ ) and lower $\mathrm{HCC}$ recurrence in the liver (OR 0.49, $\mathrm{Cl} 0.31-0.78$ ) than those who had RFA. In addition, RFA resulted in fewer adverse events (RR 4.42, $\mathrm{Cl}$ 2.74-7.15), fewer serious adverse events (RR 7.02, Cl 2.29-21.46) and shorter length of stay (mean 8.42 days, Cl 7.84-9.01).

Conclusions: RFA appears to be superior to PEI and PAI in terms of mortality. In non-surgical candidates, RFA has lower cancer-related mortality and recurrence than PEI. In surgical candidates, RFA has fewer complications and shorter length of stay but higher recurrence rates. Further high quality RCTs are required in this group. 
